\title{
Cognitive-Communication Interventions for Youth with Traumatic Brain Injury
}

\author{
Sarah A. Laane, M.S. ${ }^{1,2}$ and Lori G. Cook, Ph.D., CCC-SLP, CBIST ${ }^{1,2}$
}

\begin{abstract}
Traumatic brain injuries (TBIs) are relatively common in the pediatric population, yielding several potential challenges across a wide range of skills and abilities. Cognitive-communication disorders are particularly prevalent, with implications for long-term academic and social outcomes. While considerable evidence exists for identifying and characterizing the effects of cognitive-communication deficits, evidence informing effective interventions is still emerging. This review includes discussion of individual factors that affect treatment needs and outcomes as well as evidence that supports cognitive-communication intervention approaches at both a fundamental/discrete and integrated level. Also addressed is the need for modifying contextual factors that may be barriers as well as augmenting facilitators of successful communication and participation, including collaboration with everyday communication partners and identification and use of appropriate accommodations. Overall, research suggests a growing trend toward interventions that are individualized, dynamic, and combine multiple approaches for cognitive-communication treatment after pediatric TBI.
\end{abstract}

KEYWORDS: cognitive-communication, traumatic brain injury, intervention, pediatric

Learning Outcomes: As a result of this activity, the reader will be able to (1) explain the importance of individual differences when designing a treatment plan for cognitive-communication disorders following pediatric traumatic brain injury; (2) contrast interventions for cognitive-communication disorders that support discrete, fundamental processes versus integrated, higher-order processes; (3) describe the current trend toward interventions for cognitive-communication disorders that incorporate multiple treatment approaches, are individualized, and are dynamic in delivery over time.

${ }^{1}$ Center for BrainHealth, The University of Texas at Dallas, Dallas, Texas; ${ }^{2}$ School of Behavioral and Brain Sciences, The University of Texas at Dallas, Richardson, Texas.

Address for correspondence: Lori G. Cook, Ph.D., CCC-SLP, CBIST, Center for BrainHealth, The University of Texas at Dallas, 2200 West Mockingbird Lane, Dallas, TX 75235 (e-mail: Lori.Cook@utdallas.edu).
Current Perspectives on Speech-Language Pathology and Pediatric Traumatic Brain Injury; Guest Editor, Jennifer P. Lundine, Ph.D., CCC-SLP.

Semin Speech Lang 2020;41:183-194. Copyright (C) 2020 by Thieme Medical Publishers, Inc., 333 Seventh Avenue, New York, NY10001, USA. Tel: +1(212) 760-0888. DOI: https://doi.org/10.1055/s-0040-1701686.

ISSN 0734-0478. 
A traumatic brain injury (TBI) to the developing brain can have significant longterm consequences, thus necessitating a continuum of care, including appropriate identification, dynamic assessment, collaborative treatment, and ongoing monitoring. ${ }^{1-3}$ While there is a growing body of research available on the impact of pediatric TBI to guide evidenceinformed approaches to assessment, ${ }^{2,4,5}$ less evidence is available to inform best practices for treatment, particularly in later stages of recovery.

Due to the broad spectrum of deficits associated with TBI in youth, intervention planning involves consideration of many relevant factors. MacDonald ${ }^{6}$ demonstrates this range of needs in her model of cognitivecommunication competence, which includes seven key domains to consider for assessment and treatment of brain injury: individual, context/environment, cognition, communication, emotional, physical, and communicative competence. ${ }^{6}$ Individual factors comprise both preinjury aspects such as age, sex, education, language style, and culture, as well as injuryrelated effects such as etiology, severity, location, and extent of neurological impairment. The context/environment domain considers the communication demands (e.g., academic, occupational, social) and the child's communication partners. For example, the communication demands of a preschooler will be markedly different than those of a high school student. The preschooler likely has more inherent predictability and cues built into her interactions with teachers and parents, while a high school student is required to carry a higher cognitive load through tasks demanding greater working memory, reduced response time, more complex forms of attention, and code switching between speaking with peers and multiple teachers. Therefore, treatment should target cognitive-communication skills that are most pertinent to the needs of the individual. Beyond targeting skills needed for day-to-day interactions, research supports training communication partners to increase communicative competence in several settings, including in the school, home, and community. ${ }^{6}$

TBI can also cause physical impairments that should be considered when designing both assessment and treatment. ${ }^{6}$ Physical challenges experienced by a child with TBI might include, but are not limited to, fatigue, dizziness, sleep disorders, and hearing and vision impairments. Other possible physical conditions that are included in the wide range of possible therapy needs include motor speech disorders such as dysarthria and apraxia, affecting articulation, respiration, phonation, resonance, strength, coordination and speed of movement, and voice or prosody disorders affecting quality, loudness, pitch, and fluency. ${ }^{6}$ Additionally, the effects of emotional changes postinjury on communication and cognition should be considered during the treatment process. Emotional changes can include anxiety, stress, depression, posttraumatic stress disorder, anger, and changes to motivation and self-concept.

All communication modalities can be affected by TBI due to their interaction with cognitive, linguistic, emotional, physical, and contextual factors. ${ }^{6}$ The communication domain includes treatment considerations for auditory comprehension, verbal expression, reading comprehension, written expression, and pragmatics. The cognitive domain includes assessing and treating cognitive changes that will influence communication. When treating individuals with pediatric TBI, it is important to recall that the most common cause of communication disorders for this population is due to underlying cognitive impairments such as attention (sustaining, shifting, dividing), working memory (verbal and visuospatial), declarative learning, executive functions (e.g., abstract thinking, emotional regulation, planning), and social cognition. ${ }^{2}$ Though language disorders such as aphasia and speech disorders such as dysarthria can result from TBI, cognitive-communication disorders are the most common clinical finding. ${ }^{7}$ The cognitive processes commonly affected by TBI make up the foundation for higher-level communication skills across language domains (phonology, morphology, syntax, semantics, and pragmatics) and modalities (listening, speaking, reading, writing). ${ }^{2,5}$ For example, deficits in language comprehension may arise from decreased working memory, as a student is attempting to process a large quantity of incoming information. Deficits in declarative memory may result in reduced 
expressive vocabulary, while deficits in executive functions may lead to shorter utterance length. Both of these deficits may present as overall reductions in expressive language. Therefore, treatment for cognitive-communication disorders generally focuses on cognitive deficits that affect communication. Using a model such as the cognitive-communication competence model, ${ }^{6}$ clinicians can ensure that the full range of an individual's communication impairment is captured in a treatment plan.

Currently, treatment of pediatric TBI generally includes an interdisciplinary team working together to address these different domains to provide a holistic approach to intervention. ${ }^{8}$ Speech-language pathologists make up an important part of this treatment team during all stages of rehabilitation, from acute and inpatient to community-based interventions, in cognition and communication. ${ }^{6}$ Because the most common cause of communication disorders for this population is underlying cognitive impairments, the aim of this article is to summarize the current trends in related therapeutic interventions for children with TBI and to highlight several representative approaches.

\section{INDIVIDUAL FACTORS}

Before selecting a treatment approach, it is crucial to take into account individual factors related to the child. Multiple factors may be predictive of a child's performance and recovery, and thus serve as important considerations for selecting appropriate interventions. According to a scoping review by Cermak and colleagues, ${ }^{1}$ one such factor to consider is the severity of the TBI, which can be predictive of cognitivecommunication deficits facing the child, although it is not the sole predictor of performance. For example, Walz and colleagues ${ }^{9}$ compared the narrative discourse skills of children with moderate TBI, severe TBI, and orthopaedic injuries, and found that deficits differed between the three groups. Children with both moderate and severe TBI presented with reduced language production, less relevant story information, fewer gist propositions, reduced ability to answer explicit questions, and reduced ability to recognize unimportant facts, when compared with the orthopaedic popula- tion. Children with severe TBI also presented differently from those with moderate TBI, as those with severe TBI had significantly poorer story recall skills with poorer recognition of unimportant story details. ${ }^{9}$ Therefore, when designing treatment for a child with severe TBI, the clinician may choose to target discrete skills such as teaching story grammar and discriminating between important and unimportant information, while a clinician working with a child with a mild-to-moderate TBI may target treatment toward higher level skills such as extracting the central meaning ("gist") of a passage.

Age at the time of injury is another individual factor that should be taken into account when planning treatment. A brain injury is likely to have a more significant impact on skills that are still developing or yet to be developed, compared with skills that are already well consolidated at the time the injury is sustained. ${ }^{10}$ Cermak and colleagues ${ }^{1}$ examined nine studies regarding age of TBI and performance measures and found that, in general, narrative discourse, reading comprehension, and highlevel language skills appear most susceptible to early childhood TBI. When an intervention is provided at an appropriate developmental level for the child, outcomes are more promising for cognitive gains. ${ }^{11}$

Individual contextual factors have also been found to affect recovery and thus have implications for intervention planning. Addressing contextual factors such as family stress, school supportiveness, and personal coping styles can improve outcomes. ${ }^{12}$ For example, improved therapeutic outcomes have been found when intervention has included or taken into account (1) parent and sibling training related to environmental modifications/supports, (2) the long-term effects of a TBI, (3) typical developmental milestones, and (4) advocacy in school environment. ${ }^{12}$ Psychosocial barriers for families of lower socioeconomic backgrounds (e.g., parental unemployment, accesses to transportation, ability of school to provide appropriate supports, ability to access outpatient rehabilitation services) also increase family stress; so, connecting families to resources should be a key consideration when developing a wellrounded treatment plan. Contextual factors 
can be incorporated into treatment during any stage. Importantly, addressing contextual variables during therapy may boost the effects of treatment during the chronic stage of recovery when many patients have "plateaued" in other therapeutic areas. ${ }^{12}$

\section{APPROACHES TO INTERVENTION}

Typically, treatment approaches for cognitivecommunication disorders are categorized into four types: restorative, compensatory, habilitative, and functional/contextual. ${ }^{13}$ Restorative approaches provide direct therapy to restore or train lost functions (e.g., using a card sorting task to rebuild sustained attention). Conversely, compensatory approaches focus on capitalizing on retained abilities by implementing strategies to modify approaches to tasks. This includes providing accommodations for specific areas of deficit (e.g., short breaks during class time to compensate for decreased sustained attention). Compensatory approaches may also include the use of internal aids such as mnemonics, visual imagery, or chunking, or external aids such as calendars, checklists, timers, and headphones. A babilitative approach focuses on training and retaining skills that are developing postinjury. Lastly, a functional/contextualized approach describes a treatment that targets personally meaningful goals that can be generalized to everyday activities, such as through goal attainment scaling, an evidence-supported technique whereby both the client and clinician collaborate to create goals and rate progress. ${ }^{14}$ These approaches to intervention are by no means mutually exclusive, as treatment often includes aspects spanning more than one category.

Interventions can also be categorized based on the level of complexity of the therapeutic tasks. Some interventions support basic cognitive skills or fundamental processes using a bottom-up approach, while others support higherorder processing using a top-down approach. The Colorado Department of Education brain injury manual ${ }^{5}$ provides a framework for considering these cognitive complexities using a "building blocks" analogy. They describe fundamental processes (or basic cognitive skills) memory, attention, inhibition, sensory-motor, and processing speed—as the bottom building blocks to a pyramid. Without these fundamental processes or "building blocks" holding up the proverbial pyramid, higher-level cognitive skills will not be functional, as these lower-level skills serve as the foundation, "holding-up" the higher-level skills. Fundamental cognitive skills are often targeted using restorative, drill-based therapies. Higher-order focused interventions, in contrast, focus on the processing required for more complex skills such as reasoning, social cognition, and executive functions. These complex skills can be thought of as skills located "higher" on the cognitive pyramid because they require the functional integration of more foundational skills. Complex or higher-level skills may be bolstered through metacognitive, strategy-based, or compensatory approaches. While these treatment-approach categorizations make it easy to conceptualize different isolated treatment types, in practice, effective treatment should likely include elements of multiple approaches. For example, a drill-based short-term memory task (fundamental process), in which a patient is asked to repeat back a list of five items after a delay, can be considered restorative (with the goal of improving shortterm memory ability) but can also include a compensatory component (using higher-order processing) by coaching the patient to use a strategy such as semantic chunking (i.e., considering categorical/thematic similarities) to retain the five items.

\section{INTERVENTIONS SUPPORTING FUNDAMENTAL PROCESSES}

Interventions that support fundamental processes generally target basic cognitive skills using a bottom-up approach, including discrete cognitive skills or functions such as declarative memory, sustained attention, processing speed, or working memory. These interventions are typically implemented using drill-based approaches. Many are grounded in principles of experiencedependent neuroplasticity, ${ }^{15}$ leveraging elements of repetition, intensity, and specificity in their design to induce change. Overall, research supports the benefits of such cognitive interventions for children ${ }^{16}$; however, these methods are most beneficial for the cognitive skills directly targeted, with poor generalization to 
nontargeted tasks or functions. ${ }^{2,11,16} \mathrm{~A}$ recent systematic review and meta-analysis of pediatric cognitive interventions found that interventions that targeted attention, working memory, and short-term memory showed sizable positive improvements for specific tasks in all three domains but only small positive effects on overall behavior rating scales and academic achievement in the areas of working memory and attention. ${ }^{16}$ Several methods have been used to target fundamental processes, including drill and practice methods, direct attentional training, and errorless learning. To improve efficacy and generalization of cognitive skills targeted by these fundamental approaches, there is a trend toward integrating therapy for fundamental processes with therapy methods that are supported by higher-order processing (e.g., combining a drill and practice approach with a metacognitive approach). Additionally, interventions supporting fundamental processes are most effective when specific therapy tasks are matched to the individual needs of the child. The following sections provide examples of methods targeting fundamental processes that can be strengthened using individualized and combined approaches.

\section{Drill and Practice}

Drill and practice is the therapeutic approach of using repetitive drills to practice specific target skills. ${ }^{13}$ The theory behind this approach is that repetitive drill and practice will strengthen neural pathways through repeated activation. This technique is found to be most effective when spaced practice is employed, that is, when practice sessions are spaced out in several short sessions over a longer period of time. ${ }^{17}$

However, support for treatment models that include more elements than drill and practice comes from the lack of evidence that direct training of specific communication skills generalizes to overall communicative gains.,11 A recent systematic review of cognitive rehabilitation treatment programs for children and adolescents with acquired brain injuries suggests that multicomponent interventions have potential for the most functional benefits. ${ }^{11}$ Specifically, a metacognitive/strategy-based approach combined with a drill-based approach was more effective at treating a range of cogni- tive functions than either approach individually. Drill-based approaches were found to promote cognitive improvements, but only when outcome measures were similar to trained tasks. For example, children exposed to a computerized drill and practice program showed improvement on the verbal working memory task that was trained, but did not show significant improvement on standardized psychometric outcome measures or in academic performance. ${ }^{18}$ Metacognitive and/or strategy-based approaches alone also yielded improvements, but gains were limited to social behavior and adaptive communication behaviors. ${ }^{11}$ Additionally, metacognitive/strategybased treatment approaches yielded greater improvements in cognitive outcomes when provided in a family or peer-supported context, reinforcing the importance of a treatment model that addresses all domains of communication competencies, including environmental/contextual factors.

\section{Direct Attention Training}

Direct attention training (DAT) is an intervention that is typically considered to be a stimulus, drill-oriented approach. ${ }^{19}$ DAT uses repetitive drills to target specific areas of attention (i.e., sustained, selective, alternating, divided), with the premise that overall attentional abilities can be improved by stimulating specific areas with repetitive exercises. In recent years, the evolution of DAT provides yet another example of the trend toward combined approaches, as some recent studies advocate for combining DAT drills with a metacognitive approach. ${ }^{20,21}$ One example of an intervention using this combined approach comes from a study by Galbiati and colleagues, ${ }^{21}$ who provided an attention-specific intervention with metacognitive strategy instruction to 6- to 18-year-olds with TBI. Children in the experimental group participated in 45-minute one-on-one training sessions, four times a week, for 6 months. Sessions included both computer-based and table-top attention drills with a focus on increasing the children's awareness of their cognitive abilities. They found that the children in the experimental treatment group demonstrated significant improvements on global attention measures, as 
well as in specific attentional areas of impulsivity control, omission errors, and adaptive behavior, demonstrating that attentional training combined with metacognitive strategies generalized to other cognitive-communicative skills.

Another example of this trend comes from Sohlberg and colleagues' Attention Improvement Management (AIM) program. ${ }^{20}$ This computer-based intervention combines direct attention and working memory drills with the implementation of metacognitive strategies to improve cognitive processes impaired by TBI. Though the results from this pilot study were mixed, the study serves as another example of the trend toward combining DAT with metacognitive strategies. Furthermore, the variability of results reinforces the need for individualized treatment plans. Though AIM is a computer-based program created with the intent of decreasing the need for highly trained clinical decision making, researchers found clinical intervention necessary to override programming to adapt the training program to the individual needs of the participants during the clinical trial. ${ }^{20}$ For example, clinicians found that some participants required changes in the difficulty level of tasks to sustain attention or motivation. Clinical decision making was also used to select metacognitive strategies to fit the individual needs and preferences of participants. Modifications implemented to maximize the effectiveness of structured programs such as these demonstrate the need for dynamic and individualized interventions for children and adolescents with TBI. Computerized cognitive training programs, like DAT, which have been growing in popularity for both clinical and nonclinical use, can incorporate closed-loop models which provide real-time, quantitative feedback to facilitate dynamic adjustments. However, there is still limited evidence supporting their use over traditional cognitive treatments after pediatric brain injury. ${ }^{22}$

\section{Errorless Learning}

Errorless learning is a technique in which patients learn target skills with minimized opportunities for errors. In this approach, the clinician enhances the environment so that the patient can only produce the correct answer. ${ }^{13}$
Errorless learning is typically used with persons who have severe memory impairments and generally includes breaking tasks into discrete steps and modeling the target behavior for the individual before each step. ${ }^{17}$ An example of this could be a clinician pointing to a visual calendar and saying, "Look, today is Friday. What day is it?" The expectation is that the patient will use the provided information to respond, "Friday." Errorless learning discourages guessing and encourages immediate correction of errors ${ }^{17}$ based on the principle that eliminating interference from incorrect responses enhances learning. ${ }^{23}$ Errorless learning requires conscientious fading of supports and prompts. ${ }^{17}$

While the use of errorless learning with adults who have sustained brain injuries is widely supported, evidence for its use with children post-TBI is extremely limited, and support for its efficacy is mixed. Haslam and colleagues compared trial-and-error learning to two forms of errorless learning, traditional errorless learning (therapist provides correct answers) and self-generation errorless learning (learner produces correct response given rich cueing and corrective responses from therapist). ${ }^{24}$ Participants, aged 11 to 16 years, completed word learning trials under each condition and were tested on word recall after a 5- and 20minute delay. While typically developing children demonstrated no difference in word recall based on learning style, children with acquired brain injury demonstrated significantly improved recall for words from errorless learning conditions over the trial-and-error condition. No difference was found between traditional and self-generated errorless learning styles.

When the lens is narrowed from pediatric acquired brain injuries to TBI specifically, support for errorless learning is limited. Landis and colleagues used a within-subjects design to compare errorless learning with trial-and-error learning for memorizing declarative facts in youth specifically with TBI. ${ }^{23}$ Overall, they did not find support for the use of errorless learning over trial-and-error learning. To the contrary, they found that trial-and-error learning was more effective for initial learning of facts. Landis et $\mathrm{al}^{23}$ also found mixed results for the effectiveness of errorless learning for 
retaining information over longer periods of time. Although they were unable to identify any specific age or severity level that consistently benefited from errorless learning over an alternative learning strategy across the extended time points, some trends were noted. Of interest, although errorless learning is often recommended for more severe memory deficits, they found the children with mild, but not moderate or severe, TBI retained information over a 2day span better with errorless learning than trial-and-error learning. They also found an advantage of errorless learning for retaining information over a 7-day span, but only for younger children with mild TBI. The only time point at which errorless learning showed an advantage for children with severe TBI was at the longest retention test point ( 77 days after training), and this advantage was seen only for younger children who sustained a severe TBI. These differences again highlight the need for highly individualized approaches to intervention in pediatric TBI.

In further support of an individualized approach it is important to note that the evidence for errorless learning from Haslam and colleagues ${ }^{24}$ did not include participants younger than 11 years, while Landis and colleagues ${ }^{23}$ included children as young as 6 years, indicating that errorless learning may be less consistently beneficial for younger ages. Alternatively, the existing studies used different learning tasks (word learning vs. fact learning), indicating that the effectiveness of errorless learning may be task specific. However, more research is needed on the efficacy of errorless learning for children with TBI.

In summary, while direct methods targeting specific skills have been in practice for many years and have been shown to promote certain positive therapeutic outcomes, recent evidence points to strategies for strengthening these approaches, including accounting for individual differences when selecting and modifying treatments and incorporating multiple treatment approaches into an intervention plan. To optimize bottom-up interventions for cognitive-communication disorders, it is important to (1) individualize strategies, trajectory, and targeted skills to fit the unique needs of the child; (2) combine drill-based activities with components from other interventions such as incorporating the use of metacognitive strategies; and (3) diversify the environment of drills to include realistic contexts for better generalization. While research supports the effectiveness of these discrete cognitive interventions on the skills they target, employing bottom-up interventions alone does not appear to generalize well to broader measures of overall functioning. ${ }^{11,16}$

\section{INTERVENTIONS SUPPORTING HIGHER-ORDER PROCESSES}

Interventions that address higher-order processes more naturally combine elements of different treatment approaches, based on the nature of the cognitive processes targeted. Although interventions targeting fundamental cognitive processes/skills are typically addressed in a bottom-up fashion, such as in the drill and practice methods described earlier, treatments supporting higher-order processes often engage a more top-down, strategy-based approach. ${ }^{25}$ Evidence from cognitive neuroscience, such as brain imaging studies informing the neural correlates of executive functioning, suggests that top-down control processes are goal-oriented, voluntary/volitional (not automatic), and internally driven (not stimulus driven) cognitive operations. ${ }^{25,26}$ To address these higher-order processes, treatments should aim to equip the individual with tools that can be employed repeatedly across dynamic task demands and real-life contexts. Through active collaboration, clinicians can help the child identify and utilize cognitive strategies to manage and perform daily tasks more effectively. To promote effective use of cognitive strategies, a therapeutic task must embed the strategy in meaningful academic or social contexts. To promote longterm generalization, practice must be intensive and ongoing, with the individual encountering multiple opportunities to use the strategy. Effective treatment must also address an individual's motivation, knowledge base, environment, and other critical factors, and many aspects must be built into the intervention to support generalization and maintenance for functional use. Beyond direct intervention with the child, it is particularly important in a strategy-based approach to engage everyday communication 
partners for carryover, when possible. Further, home program activities can engage parents to review and reinforce learned strategies with their child.

\section{Metacognitive Skills Training}

One cognitive-communication intervention category representing a strategy-based approach is the training of metacognitive strategies or behaviors that facilitate efficient allocation of cognitive resources and enhanced self-regulation, commonly utilized for individuals who exhibit difficulties with real-life executive functioning. Metacognitive skills training may involve teaching students to regulate their own behavior by breaking complex tasks into steps while thinking strategically, ${ }^{27}$ which can be taught while working on a child's schoolwork as well as other everyday activities. These can include strategies which are engaged before a task is initiated (i.e., pre-task, such as goal setting), while a task is being done (i.e., on-task, such as checking progress), and/or upon completion of a task (i.e., post-task, such as review). Metacognitive strategies might include mental imagery, repeating/clarifying instructions, predicting task difficulty, taking a break, internal self-talk, external self-talk, and rewarding self. One example spanning several of these categories is training a child to use the "Goal-Plan-Do-Review" strategy, ${ }^{28}$ designed to address difficulties with self-regulation, or the metacognitive ability to formulate, enact, and monitor a plan of action in adherence with a goal. ${ }^{29,30}$ Such an approach is designed to facilitate a top-down, or "goal-driven" action/ response and mitigate over-reliance on bottomup, or "stimulus-bound" actions/responses. For example, a child engaging in a top-down approach while executing a complex task would reference his or her goal and plan to guide the next action, whereas a child using a bottom-up approach may determine the next action/response based on immediate environmental stimuli such as the objects present. Promoting selfdriven, goal-directed action bears important implications for combating real-life breakdowns in executive functioning. ${ }^{31}$ Specifically, the Goal-Plan-Do-Review strategy provides a universally applicable, systematic tool for defining a goal, organization/planning, action initiation, self-monitoring/evaluation, and cognitive flexibility. Other metacognitive training tools include strategies for situational and selfawareness, whole body listening, and future thinking (such as putting on your "future glasses"). ${ }^{32}$

\section{Strategic Learning Intervention}

Beyond applications to enhance metacognitive skills, such as in the context of addressing executive functioning, research has demonstrated that top-down, strategy-based approaches can also be utilized to enhance learning processes for youth with brain injury. Because pediatric TBI can have a significant impact on new learning, a forward-thinking approach is crucial in supporting students to take on escalating academic demands, particularly for key transitions such as from elementary to middle school or middle to high school. One such tool is strategic learning intervention, currently exemplified by Strategic Memory Advanced Reasoning Training (SMART), developed by Chapman and colleagues as a topdown, integrated approach which provides students with cognitive strategies to more effectively evaluate, manage, synthesize, and apply information. $^{33,34}$ The efficacy of SMART has been previously evaluated across youth and adults, including evidence in both healthy/ typically developing and clinical populations. ${ }^{33}$ Specifically, for adolescents with TBI at later stages postinjury (i.e., at least 6 months postinjury), a randomized controlled study of SMART versus an active, bottom-up memory strategy training revealed significant gains for individuals in the SMART training group. In particular, participants receiving SMART improved in their ability to perform abstract thinking, as well as improving in detail/fact recall and on standardized measures of executive function (i.e., working memory and inhibition). Individuals in the control group who trained with rote memorization strategies (e.g., flash cards, mnemonics, and visual associations) showed no significant improvements in any of the cognitive performance domains evaluated. ${ }^{34}$ Overall, findings support the idea that facilitating top-down processing of information may also positively impact fundamental, bottom-up 
processes (such as improved ability to recall important facts) and further generalize to cognitive control processes (such as working memory, inhibition, and cognitive flexibility) engaged during strategic learning.

Strategic learning intervention was informed and supported by seminal research in cognitive science which has revealed that the brain is better at extracting central meaning than storing details, in that memory for "gist" is more resilient than memory for specific details. ${ }^{35,36}$ Furthermore, the ability to form bigger ideas while suppressing irrelevant details can help one think faster and more efficiently when engaged in complex information processing (i.e., thinking smarter, not harder). By equipping students who experienced TBI with strategies to engage in top-down thinking, particularly when faced with discourse-level information such as class lectures and academic texts, they are less likely to "get lost in the details and miss the big picture." Moreover, consolidating and synthesizing new information can become more manageable, thus potentially mitigating feelings of "information overload" due to strained cognitive resources. ${ }^{37}$ For example, in this program the first SMART strategy trained promotes discrimination between important and unimportant/less relevant information, with intentional blocking (e.g., crossing out) of the unimportant information before focusing on key points. Consider this approach an alternative to the standard "highlighter" approach. See Fig. 1 for a description of each of the SMART strategies.

For younger learners, strategic learning can be stimulated through activities promoting identification and discussion of main ideas/ concepts rather than focusing on detail-level recall. Strategic learning can also be supported through creative games or crafting activities involving identifying, following, and/or relaying the main or most critical steps/directions. ${ }^{38}$

To further optimize the top-down learning process, it is important to (1) use questions and other techniques to increase metacognitive awareness; (2) push for depth of understanding over breadth of recall; (3) promote integration of knowledge-connecting new information to one's own knowledge/experiences; (4) collaboratively plan and create opportunities for transfer, generalization, and application of what is learned; and (5) encourage students to connect seemingly unrelated subjects and ideas and derive their own questions. These techniques can be trained and implemented across communicative contexts and partners.

\section{MODIFYING THE COMIMUNICATION ENVIRONMENT}

In general, when engaging with the child's everyday communication partners (e.g., parents, teachers), it is important to identify and

\begin{tabular}{|c|c|}
\hline Blocking & unimportant information before focusing on key points \\
\hline $\begin{array}{l}\text { Organizing \& } \\
\text { Managing }\end{array}$ & information by chunking and labeling similar ideas by theme \\
\hline Inferencing & to extract the deeper or underlying meaning of information \\
\hline Paraphrasing & key points rather than getting bogged down in word-for-word details \\
\hline Summarizing & information by combining details together into gist-based concepts \\
\hline Abstracting & bigger-picture ideas from multiple perspectives \\
\hline $\begin{array}{l}\text { Connecting \& } \\
\text { Applying }\end{array}$ & new knowledge to your life by creating "take-home" messages \\
\hline Demonstrating & $\begin{array}{l}\text { more complete understanding by "zooming out" and "zooming in" to } \\
\text { show both the "forest" and the "trees" }\end{array}$ \\
\hline
\end{tabular}

Figure 1 Descriptions of SMART strategies. 
promote potential changes outside of the child (e.g., external strategies) that can maximize his or her cognitive-communication success. This first necessitates observing the child's behaviors in context and describing the problem (e.g., child keeps asking the same questions over and over again). One can then better understand how to relate the observed behavior to an underlying cognitive-communication impairment (e.g., how it may be related to a cognitive skill, such as poor memory). When a child is having difficulty, support needs to be provided at that moment-family members, teachers, and friends need an arsenal of strategies to guide interactions in positive directions. Key communication partners need to recognize their own interaction style and the effect that style may have on the child, taking steps to bring about change in the child's performance. Ultimately, the goal is to guide communication partners in identifying and modifying their manner and style in response to the child's everchanging strengths and needs. In addition to providing a better understanding of the child's cognitive strengths and weaknesses, a clinician can deliver ongoing coaching in cognitive skill development and improved teaching approaches or instructional strategies more sensitive to the child's current profile of cognitive abilities. Other helpful changes may include use of external supports (e.g., posted reminders/visual cues, removal of distractions). The clinician should work with caregivers to consider how to best facilitate opportunities for dynamic, interactive engagement, including permitting adequate response time, arranging the physical environment to support effective communication, reinforcing the student's communication attempts, making the student aware of others' responses, and welcoming discussion of frustrations, concerns, difficulties, and successes to inform and promote both self-insight and self-advocacy. ${ }^{3}$

\section{CONCLUSION}

Cognitive-communication abilities are not only highly interrelated but also multifaceted and dynamic in terms of development and demand. Therefore, in working with students with TBI, it is important for clinicians to think "deep and wide" to best support and equip students with the skills needed for cognitive-communication success across their ongoing recovery, development, and academic pursuits. As we have discussed, clinicians must consider several important questions during cognitive-communication treatment planning for an individual student. These questions span academic, motivational, situational, and personal factors. Clinicians should also strive to engage, empower, and equip the child's everyday communication partners, particularly the family and teachers. Ultimately, an individualized, functional, dynamic approach to cognitive-communication intervention is key to promoting global gains.

\section{DISCLOSURES}

S.A.L. has no financial or nonfinancial interests to disclose.

L.G.C. has no financial or nonfinancial interests to disclose.

\section{CONFLICT OF INTEREST}

None.

\section{REFERENCES}

1. Cermak CA, Scratch SE, Reed NP, Bradley K, Quinn de Launay KL, Beal DS. Cognitive communication impairments in children with traumatic brain injury: a scoping review. J Head Trauma Rehabil 2019;34(02):E13-E20

2. Turkstra LS, Politis AM, Forsyth R. Cognitivecommunication disorders in children with traumatic brain injury. Dev Med Child Neurol 2015;57 (03):217-222

3. DePompei R, Blosser JL. Pediatric Traumatic Brain Injury: Proactive Intervention. 3rd ed. San Diego, CA: Plural Publishing, Inc; 2019

4. McDonald S, Togher L, Code C. Social and Communication Disorders Following Traumatic Brain Injury. 2nd ed. London: Psychology Press; 2014

5. Dettmer J, Dise-Lewis JE, Colella PW, et al. Brain Injury in Children and Youth - A Manual for Educators. Colorado Department of Education; 2018. Available at: https://www.cde.state.co.us/ cdesped/tbi_manual_braininjury. Accessed $\mathrm{Au}-$ gust 21, 2019

6. MacDonald S. Introducing the model of cognitivecommunication competence: a model to guide evidence-based communication interventions after brain injury. Brain Inj 2017;31(13-14):1760-1780 
7. MacDonald S. Thinking before speaking, understanding the meaning, and reading context cues: An update on communication interventions after ABI. International Brain Injury Association. Available at: https://www.internationalbrain.org/articles/ thinking-before-speaking-understanding-themeaning-and-reading-context-cues/. Published December 10, 2012. Accessed July 26, 2019

8. Catroppa C, Anderson V. Pediatric TBI: challenges for treatment and rehabilitation. Pediatric. In: Anderson V, Yeates KO, eds. Traumatic Brain Injury: New Frontiers in Clinical and Translational Research. 5th ed. Cambridge: Cambridge University Press; 2010:192-205

9. Walz NC, Yeates KO, Taylor HG, Stancin T, Wade SL. Emerging narrative discourse skills 18 months after traumatic brain injury in early childhood. J Neuropsychol 2012;6(02):143-160

10. Chapman SB. Neurocognitive stall, a paradox in long term recovery from pediatric brain injury. Brain Inj Prof. 2006;3:10-13

11. Resch C, Rosema S, Hurks P, de Kloet A, van Heugten C. Searching for effective components of cognitive rehabilitation for children and adolescents with acquired brain injury: a systematic review. Brain Inj 2018;32(06):679-692

12. Ciccia AH, Threats T. Role of contextual factors in the rehabilitation of adolescent survivors of traumatic brain injury: emerging concepts identified through modified narrative review. Int J Lang Commun Disord 2015;50(04):436-451

13. American Speech-Language Hearing Association. Pediatric traumatic brain injury American Speechlanguage Hearing Association (Practice Portal). Available at: https://www.asha.org /PRPSpecific Topic.aspx?folderid $=8589942939$ \&section $=$ Treatment\#Treatment_Options. Accessed September 23, 2019

14. Grant M, Ponsford J. Goal attainment scaling in brain injury rehabilitation: strengths, limitations and recommendations for future applications. Neuropsychol Rehabil 2014;24(05):661-677

15. Kleim JA, Jones TA. Principles of experiencedependent neural plasticity: implications for rehabilitation after brain damage. J Speech Lang Hear Res 2008;51(01):S225-S239

16. Robinson KE, Kaizar E, Catroppa C, Godfrey C, Yeates KO. Systematic review and meta-analysis of cognitive interventions for children with central nervous system disorders and neurodevelopmental disorders. J Pediatr Psychol 2014;39(08):846-865

17. Sohlberg MM, Ehlhardt L, Kennedy M. Instructional techniques in cognitive rehabilitation: a preliminary report. Semin Speech Lang 2005;26 (04):268-279

18. Eve M, O'Keeffe F, Jhuty S, Ganesan V, Brown G, Murphy T. Computerized working-memory training for children following arterial ischemic stroke: a pilot study with long-term follow-up. Appl Neuropsychol Child 2016;5(04):273-282

19. Sohlberg MM, Avery J, Kennedy M, et al. Practice guidelines for direct attention training. J Med Speech-Lang Pathol 2003;1(03):xix-xxxix. Available at: https://link.gale.com/apps/doc/A108790948/ HRCA?u $=$ txshracd $2602 \&$ sid $=$ HRCA\&xid $=$ 22a36913. Accessed September 23, 2019

20. Sohlberg MM, Harn B, MacPherson H, Wade SL. A pilot study evaluating attention and strategy training following pediatric traumatic brain injury. Clin Pract Pediatr Psychol 2014;2(03): 263-280

21. Galbiati S, Recla M, Pastore V, et al. Attention remediation following traumatic brain injury in childhood and adolescence. Neuropsychology 2009;23(01):40-49

22. Lundine JP. Memory restoration using computerized cognitive training after pediatric traumatic brain injury: a review of the evidence. EBP Briefs 2017;12(03):1-7

23. Landis J, Hanten G, Levin HS, et al. Evaluation of the errorless learning technique in children with traumatic brain injury. Arch Phys Med Rehabil 2006;87(06):799-805

24. Haslam C, Bazen-Peters C, Wright I. Errorless learning improves memory performance in children with acquired brain injury: a controlled comparison of standard and self-generation techniques. Neuropsychol Rehabil 2012;22(05):697-715

25. Chen AJ, D'Esposito M. Traumatic brain injury: from bench to bedside [corrected] to society. Neuron 2010;66(01):11-14

26. Kane MJ, Engle RW. The role of prefrontal cortex in working-memory capacity, executive attention, and general fluid intelligence: an individual-differences perspective. Psychon Bull Rev 2002;9(04): 637-671

27. Kennedy MRT, Coelho C, Turkstra L, et al. Intervention for executive functions after traumatic brain injury: a systematic review, meta-analysis and clinical recommendations. Neuropsychol Rehabil 2008;18(03):257-299

28. Feeney T, Ylvisaker M. Context-sensitive cognitive-behavioural supports for young children with TBI: a replication study. Brain Inj 2006;20(06): 629-645

29. Wehmeyer ML, Agran M, Hughes C. Teaching Self-Determination to Students with Disabilities: Basic Skills for Successful Transition. Baltimore, MD: Paul H. Brookes Publishing; 1998

30. Ylvisaker M, Feeney T. Executive functions, selfregulation, and learned optimism in paediatric rehabilitation: a review and implications for intervention. Pediatr Rehabil 2002;5(02):51-70

31. Cook LG, Chapman SB, Levin HS. Self-regulation abilities in children with severe traumatic brain injury: a preliminary investigation of 
naturalistic action. NeuroRehabilitation 2008;23 (06):467-475

32. Ward S, Jacobsen K. A clinical model for developing executive function skills. Perspect Lang Learn Educ 2014;21:72-84

33. Chapman SB, Mudar RA. Enhancement of cognitive and neural functions through complex reasoning training: evidence from normal and clinical populations. Front Syst Neurosci 2014;8:69

34. Cook LG, Chapman SB, Elliott AC, Evenson NN, Vinton K. Cognitive gains from gist reasoning training in adolescents with chronic-stage traumatic brain injury. Front Neurol 2014;5:87
35. Brainerd CJ, Reyna VF. Fuzzy-trace theory and children's false memories. J Exp Child Psychol 1998;71(02):81-129

36. Gabrieli JDE. Memory: Pandora's hippocampus? Cerebrum 2004;6(04):39-48

37. Vogel EK, McCollough AW, Machizawa MG. Neural measures reveal individual differences in controlling access to working memory. Nature 2005;438(7067):500-503

38. Cook LG, DePompei R, Chapman SB. Cognitive communication challenges in TBI: Assessment and intervention in the long term. Perspect Neurophysiol Neurogenic Speech Lang Disord 2011;21(01):33-42 